\title{
Le traitement de l'ostéosarcome en 1992
}

\author{
S.R. Babin ${ }^{1}$ et A. Babin-Boilletot ${ }^{2}$ \\ ${ }^{1}$ Département d'Orthopédie et de Traumatologie, ${ }^{2}$ Service d'Onco-Hématologie, Hôpital de Hautepierre, F-67098 Strasbourg Cedex
}

Le traitement de l'ostéosarcome est, en 1992, pluridisciplinaire, et les progrès réalisés depuis 20 ans sont considérables. La prise en charge du malade est à la fois chimiothérapique et chirurgicale et ces deux méthodes sont actuellement indissociables. On connaît la chimiosensibilité de cette tumeur depuis les années 1970, date des premiers essais de JAFFE avec le méthotrexate à haute dose. D'autres drogues sont également efficaces comme l'adriamycine, le cyclophosphamide, l'ifosfamide et les dérivés des sels de platine. Donnée après la chirurgie, comme elle l'était dans les années 1975, la polychimiothérapie avait déjà permis d'obtenir un taux de survie en rémission complète à 5 ans de l'ordre de $50 \%$. Dès 1978, Rosen, considérant que l'ostéosarcome était d'emblée une maladie micrométastatique chez $80 \%$ des patients, et ayant montré antérieurement l'efficacité d'une chimiothérapie adjuvante, débuta un essai à New York de chimiothérapie néo-adjuvante, c'est-à-dire administrée avant tout geste chirurgical local autre que la biopsie. L'évaluation histologique de la nécrose tumorale obtenue par cette chimiothérapie première se révéla être un important facteur de pronostic: les patients étaient alors classés en «bons répondeurs》 et «mauvais répondeurs » selon que la nécrose tumorale était supérieure ou inférieure à $90 \%$ sur la pièce opératoire d'exérèse. Après le geste chirurgical local, une chimiothérapie était donnée à nouveau semblable pour tous les malades à celle qui avait été donnée avant la chirurgie dans la première étude $\mathrm{T} 7$, puis, dans l'étude suivante T 10, adaptée selon la réponse tumorale. Les résultats de ces études, actualisés récemment, montrent un taux de survie sans récidive voisin de $70 \%$ à 10 ans. Toutefois, les résultats des malades «mauvais répondeurs» sont inférieurs à ceux des «bons répondeurs ». L'esprit du traitement néoadjuvant était dès lors acquis et en 1992, il est communément admis. Ses modalités sont variables selon les équipes et font dans certains cas appel à d'autres modes d'administration de la chimiothérapie comme la voie intraartérielle utilisée par Jaffe. On peut alors utiliser de l'adriamycine ou du cisplatinum. Toutefois ce mode d'administration, s'il est efficace sur la tumeur primitive, ne semble pas diffuser à distance aussi bien, et l'action sur le développement des métastases est, dans certaines mains, nettement moindre. La plupart des équipes utilisent actuellement des schémas d'administration intraveineuse. En sus du traitement précoce des micrométastases, l'utilisation de la chimiothérapie néo-adjuvante permet dans certains cas, en limitant l'extension de la tumeur primitive, une intervention locale dans de meilleures conditions. Toutefois, les métastases tardives au-delà de 5 ans existent, dans un petit nombre de cas, même chez les malades « bons répondeurs » au départ.

Sur le plan du traitement chirurgical local, l'approche biologique de la reconstruction par allogreffe est adoptée par de nombreuses équipes; cependant les auteurs s'accordent pour reconnaître que le taux de complications est élevé, qu'il s'agisse d'infection, d'absence de fusion, de fracture de fatigue, d'instabilité ou de détérioration du cartilage. R.S. Bell, A. Davis et coll de Toronto, obtiennent les meilleurs résultats avec les allogreffes intercalaires alors que le pourcentage d'échecs concernant les arthrodèses avec allogreffes, les prothèses manchonnées et les allogreffes ostéo-articulaires se situe autour de $50 \%$. S'agissant des allogreffes ostéo-articulaires des deux versants du genou, M.G. Rock, E.Y.S. Chao et coll de la Mayo Clinic évaluent les échecs entre 40 et $50 \%$ et pensent qu'il est préférable de recourir aux endoprothèses de reconstruction dans les localisations fémorales distales; pour ce qui est du tibia proximal, ils voient dans l'allogreffe ostéo-articulaire ou dans la prothèse manchonnée la solution à la restauration de l'appareil extenseur du genou. La reconstruction par mégaprothèse associée au traitement des parties molles au moyen de la plastie du jumeau interne défendue par $J$. Dubousset et G. Missenard de l'hôpital Saint-Vincent-de-Paul (Paris) représente une alternative valable. Dans le but d'améliorer les conditions de fusion entre l'hôte et l'allogreffe un certain nombre de promoteurs proposent d'adjoindre à l'allogreffe une greffe autogène de péroné vascularisé. $R$. Capanna et M. Manfrini, de l'institut Rizzoli de Bologne utilisent ce procédé dans les reconstructions intercalaires des localisations métaphyso-diaphysaires et leurs résultats préliminaires à court terme sont encourageants. M.J. O'Connor, M.G. Rock et coll de la Mayo Clinic recommandent cette technique dans les arthrodèses de l'épaule après résection tumorale nécessitant le sacrifice des abducteurs. C.M. Clinkscales, M.B. Woodf et H. Sim de la Mayo Clinic appliquent ce principe 
dans le traitement des échecs de reconstruction par allogreffe en cas d'infection ou de pseudarthrose. La technique de reconstruction de l'anneau pelvien imaginée par J. Puget et G. Utheza de Toulouse est intéressante parce qu'elle s'adresse non pas à l'allogreffe mais à l'autogreffe que représente l'extrémité proximale du fémur au niveau de laquelle peut être modelée une cavité cotylö̈dienne et scellée une prothèse totale de hanche. La prothèse-selle de E. Nieder (Hambourg) initialement conçue pour les reprises de prothèse totale de hanche avec destruction du cotyle, est susceptible de rendre service après certaines résections partielles du bassin quand on décide de ne pas recourir à l'allogreffe. L'approche mécanique de la reconstruction par endoprothèse métallique pose, outre la question des complications liées aux impératifs de la chirurgie carcinologique et aux contraintes de la chimiothérapie, celle de la longévité. Sur le plan expérimental, J. Hua et P.S. Walker de Stanmore montrent que la distribution des contraintes est meilleure pour les queues non cimentées que pour les queues cimentées, que leur implantation soit proximale ou distale. Cette répartition plus proche de la normale avec les queues non cimentées devrait concourir à diminuer le remodelage osseux indésirable observé au niveau de la jonction et par conséquent à augmenter la longévité de la tenue de l'implant dans l'os. Sur le plan clinique, le recul des prothèses cimentées est plus long que celui des prothèses non cimentées. P.S. Unwin, J.P. Cobb et P.S. Walker ont pu étudier sur une période de 30 ans le devenir de 221 prothèses cimentées fémorales proximales et de 447 prothèses fémorales distales de reconstruction après résection pour tumeurs. Ils évaluent à $82 \%$ la probabilité de survie sans échec à 10 ans d'une prothèse fémorale proximale et à $61 \%$ celle d'une prothèse fémorale distale, l'échec étant défini comme la nécessité de réopérer ou d'amputer pour une autre raison que la récidive locale.

Malgré ces progrès indéniables, les solutions à un certain nombre de questions restent en suspens. Indépendamment du difficile problème de reconstruction après résection chez l'enfant, la solution prometteuse de l'allogreffe a fait apparaitre chez l'adulte un taux significativement élevé de complications. Des connaissances approfondies en immunologie, les procédés qui visent à favoriser la revascularisation aideront peut-être à réduire la fréquence des échecs. Pour ce qui est des prothèses, leur longévité constitue un pari sur l'avenir où le principe du «sans ciment» et le système modulaire devraient être des atouts de poids. Le traitement général de la maladie par chimiothérapie n'a pas encore résolu tous les problèmes de rechutes ou de métastases: certains malades ne tolèrent pas les doses utiles de la chimiothérapie et il faut donc adapter les drogues et leur dosage; en particulier grâce à un apport d'étude pharmacocinétique individuel. Surtout, certaines tumeurs sont résistantes à la chimiothérapie d'emblée et les drogues actuellement utilisées de façon standard chez ces malades sont insuffisantes pour entraîner une réponse appréciable. L'étude in vitro sur la tumeur de la présence des gènes de multirésistance (MDR 1 ou MDR $2=$ multidrug resistance 1 ou 2) permettra peut-être d'utiliser d'emblée les drogues efficaces. Enfin, l'étude des oncogènes spécifiques et de leur amplification pourrait dans un avenir proche permettre d'étudier certains facteurs pronostiques biologiques et d'adapter plus tôt le traitement chez les patients résistants ou rapidement évolutifs.

On trouvera les sources bibliographiques dans :

1. Complications of limb salvage; prevention, management and outcome. KLB Brown (ed) 6 th International Symposium on Limb Salvage, Montreal, September 8-11, 1991. ISOLS Montreal

2. Clinical Orthopedics and Related Research, 270, September 1991 